\title{
Multiple-Access Interference-Resistant Acquisition for Band-Limited CDMA Systems with Random Sequences
}

\author{
Ashok Mantravadi and Venugopal V. Veeravalli, Senior Member, IEEE
}

\begin{abstract}
The problem of estimating the propagation delay of a new user in a coded band-limited DS/CDMA system in the presence of multiple access interference (MAI) is considered. MAI-resistant acquisition schemes are developed for a general CDMA system without the constraint that the spreading sequences of the users repeat every symbol period. It is assumed that the spreading sequences and delays of the interfering users are known. However, knowledge of their amplitudes, which would need estimation, is not assumed, and their unreliable code-symbol estimates are not used. Under this scenario, acquisition schemes are derived based on the maximum-likelihood (ML) criterion. The performance of an approximation to the ML scheme is analyzed using Gaussian approximations and by assuming that the chip boundaries of the new user are known a priori. Simulations show that the analysis is reasonably accurate for parameters in the realm of practical interest.
\end{abstract}

Index Terms-Band-limited signals, code division multi-access, interference rejection, maximum-likelihood estimation, propagation delay estimation.

\section{INTRODUCTION}

$\mathbf{I}$ N HIGH capacity CDMA systems, the acquisition process is limited by the multiple-access interference (MAI) from other users. Traditional approaches to acquisition in the presence of MAI have treated the MAI as additive random noise without exploiting any of the structure in the interference. Clearly, acquisition schemes can be improved considerably if the MAI can be cancelled or suppressed. This has motivated recent work on MAI resistant acquisition techniques. Examples include subspace-based decomposition techniques [1], [2], maximum-likelihood techniques involving sample statistics [3], [4], and joint MMSE acquisition and detection [5], [6]. The underlying assumption in all of this work is that the spreading sequences of the users repeat every symbol period (short sequences). But short sequences may be impractical for asynchronous (wireless) CDMA systems. Short sequences allow for the possibility that two (or more) users have signals that are highly correlated over several bit intervals - this reduces the

Manuscript received July 30, 1999; revised November 29, 1999. This work was supported in part by the NSF under Grant NCR-9523967.

A. Mantravadi is with the School of Electrical Engineering, Cornell University, Ithaca, NY 14853 USA (e-mail: ashok@ee.cornell.edu).

V. V. Veeravalli was with the School of Electrical Engineering, Cornell University, Ithaca, NY 14853 USA. He is now with the University of Illinois at Urbana-Champaign, Urbana, IL 61801 USA.

Publisher Item Identifier S 0733-8716(00)05290-2. worst case performance and reuse efficiency in asynchronous CDMA systems [7]-[9].

Practical wireless CDMA systems, such as those specified in the IS-95 standard [10] and the CDMA 2000 proposal [11], randomize the users' signals on the reverse (asynchronous) link by using spreading sequences whose periods are much greater than the processing gain. Such systems are sometimes referred to as R-CDMA (Random-CDMA) systems [7]. It is of interest to develop acquisition schemes that can mitigate MAI in R-CDMA systems. Previous work on MAI-resistant acquisition for R-CDMA systems has been based on an interference cancellation approach [12]. The main drawback of this approach is that interference cancellation requires precise knowledge of the interferers' spreading sequences, delays, amplitudes, and code symbols. Consider the situation where a single ${ }^{1}$ new user is to be acquired in the presence of interference from existing users in the system who have already been acquired and are being successfully demodulated. Here, it is reasonable to assume that the interferers' spreading sequences are known at the receiver, and that accurate delay estimates are available. However, it may not be reasonable to assume that accurate amplitude estimates are available, particularly for wireless channels. Furthermore, code symbol estimates at the outputs of the detector are unreliable if low rate error control coding is used, unless we assume an impractical scheme where the code symbols of all the interferers are reconstructed from the decoded bits. Hence, it is of interest to develop MAI-resistant acquisition schemes for R-CDMA systems that assume knowledge only of the interferers' spreading sequences and delays.

In this paper, we develop and analyze such MAI-resistant acquisition schemes. The approach closely resembles that used in developing the decorrelating multiuser detector [13] —we decorrelate the interference (linearly) before estimating the intended user's parameters. A similar approach, albeit for joint delay estimation of all the users, was also investigated in [14]. The computational complexity of the acquisition scheme is of the order of that required (per user) for a decorrelating detector involving the same number of users. However, while decorrelating detection may be impractical for R-CDMA systems, since the decorrelating matrix needs to be updated from symbol to symbol at the coded data rate, decorrelating acquisition of a single user with a sufficiently long preamble does not have the same timing constraints and should be practical.

\footnotetext{
${ }^{1}$ A situation may arise where more than one user enters the system at the same time. In this case, we can assume that the users are acquired one at a time.
} 


\section{SYSTEM MODEL}

We consider a DS/CDMA model with $K$ users, where the received complex baseband signal is given by

$$
\begin{gathered}
r(t)=\sum_{k=1}^{K} \sum_{n=-1}^{M-1} \sqrt{\frac{2 \mathcal{E}_{k}}{N}} b_{k, n} c_{k}^{(n)}\left(t-\tau_{k}\right) e^{j \phi_{k}}+w(t), \\
t \in\left[0, M T_{s}\right]
\end{gathered}
$$

and where the following hold.

- $b_{k, n}$ is symbol $n$ of user $k$, and $T_{s}$ is the symbol period.

- $c_{k}^{(n)}(t)=\sum_{j=0}^{N-1} c_{k, j}^{(n)} \psi\left(t-n T_{s}-j T_{c}\right)$. Here $T_{c}$ is the chip period, $\psi(t)$ is the unit-energy chip waveform, $c_{k, j}^{(n)}$ is the (complex) chip sequence, and $N=T_{s} / T_{c}$ is the processing gain of the system.

- $\phi_{k}, \tau_{k}$, and $\mathcal{E}_{k}$ are, respectively, the carrier phase offset, delay and the received symbol energy of the user $k$. Define $A_{k}=\sqrt{2 \mathcal{E}_{k} / N}$ to be the corresponding received amplitude.

- $w(t)$ is a zero mean circularly complex Gaussian process with two-sided power spectral density $N_{0}$, i.e., $R_{w}(\tau)=$ $(1 / 2) E\left[w^{*}(t) w(t+\tau)\right]=N_{0} \delta(\tau)$.

Thus, we are considering an $M$-symbol observation of the received signal. In a synchronous situation, the symbols would be indexed from $n=0$ to $n=M-1$. However, since the users are asynchronous, there is an additional symbol corresponding to $n=-1$ in the observation window. Also, we have assumed a signal model where the users undergo flat fading, and the symbol energy above includes the fading effect. The fade levels of all the users are assumed to remain constant over the observation window. ${ }^{2}$ The flat-fading assumption is in general restrictive, but may be applicable in some indoor wireless scenarios. For example, with a typical indoor delay spread of $100 \mathrm{~ns}$, a $1.25 \mathrm{MHz}$ CDMA signal would undergo flat fading. Extension to the frequency-selective fading channels is under consideration in a separate paper.

The new user entering the system is taken to be the one with $k=1$. In addition, we assume the following.

- The initial timing uncertainty is $L$ chips, i.e., $\tau_{1} \in\left[0, L T_{c}\right)$. Then, we can write $\tau_{1} / T_{c}=n_{1}+\alpha_{1}$, with $n_{1} \in\{0,1 \cdots L-1\}$ and $\alpha_{1} \in[0,1)$.

- The new user transmits a preamble with known symbols over the observation interval. Since we consider long spreading sequences, there is no loss of generality in assuming that $b_{1, n}=1, \forall n$.

- We assume initially that we have coherent demodulation and perfect carrier synchronization with respect to user 1 , so that $\phi_{1}=0$. While current CDMA systems do not allow for carrier phase estimation before delay acquisition on the reverse link, future standards [11] may provide a pilot channel to help in initial acquisition, and the coherent demodulation assumption will be valid in such a scenario. We consider extensions to the noncoherent case in Section IV.

\footnotetext{
${ }^{2}$ This assumption can actually be relaxed to have the fade levels of the interfering users be different over different symbols.
}

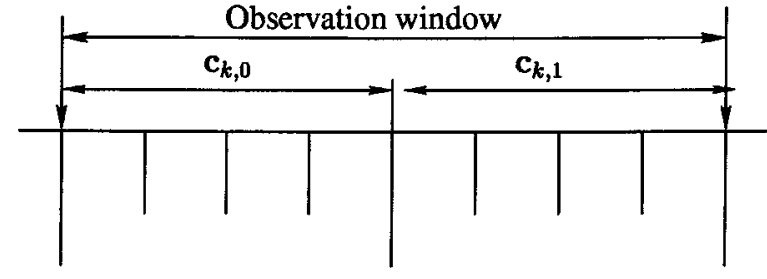

a. Symbol synchronous

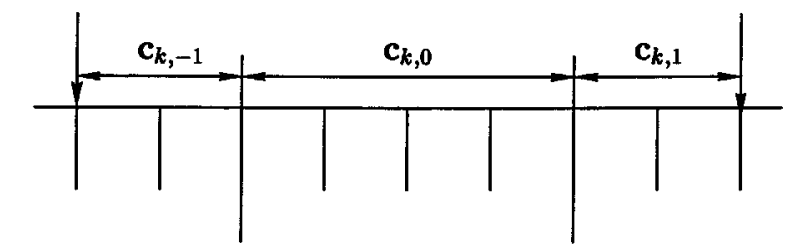

b. Chip synchronous

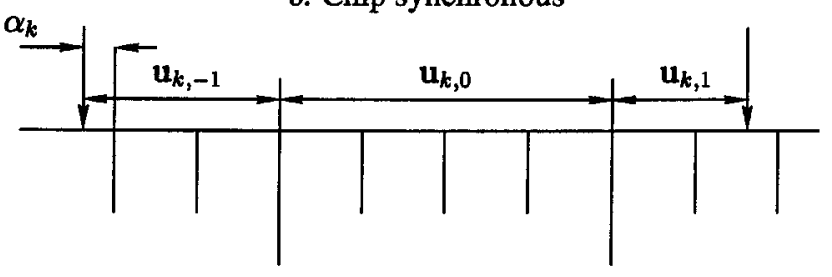

c. Chip asynchronous

Fig. 1. Illustration of model with effective users: $M=2$. (a) Symbol synchronous. (b) Chip synchronous. (c) Chip asynchronous.

- For $k \neq 1, \tau_{k} \in\left[0, T_{s}\right)$, and hence $\tau_{k}$ can be written as $\tau_{k}=\left(n_{k}+\alpha_{k}\right) T_{c}$, with $n_{k} \in\{0,1 \cdots N-1\}$ and $\alpha_{k} \in$ $[0,1)$. Since the sequences of the other users are known, these delays affect only the interfering symbol patterns and there is no loss of generality in assuming that $\tau_{k} \in$ $\left[0, T_{s}\right)$.

Under the above assumptions, the received signal of interest reduces to

$$
\begin{aligned}
r(t)= & A_{1} c_{1}\left(t-\tau_{1}\right)+\sum_{k=2}^{K} \sum_{n=-1}^{M-1} \\
& \cdot A_{k} b_{k, n} c_{k}^{(n)}\left(t-\tau_{k}\right) e^{j \phi_{k}}+w(t) .
\end{aligned}
$$

For further analysis, this model can be converted to one where the signals corresponding to each symbol of each user occurring in the observation interval are identified as different (effective) users, as shown ${ }^{3}$ in Fig. 1. Since we allow the chip waveform to have a width greater than $T_{c}$, the number of effective users corresponding to each user would in general depend on the width of the chip waveform. However, we assume that this width is much smaller than the symbol interval, so that the number of effective users can be considered to be just $M+1$ per user. Finally, since user 1 employs a preamble, we have a model involving $K_{\text {eff }}=(K-1)(M+1)+1$ effective users. We define $\tilde{c}_{1}(t)=c_{1}\left(t-\tau_{1}\right)$ and, for $k=2, \cdots, K$ and $0 \leq t \leq M T_{s}$

$$
\tilde{c}_{(M+1)(k-2)+n+2}(t)=c_{k}^{(n-1)}\left(t-\tau_{k}\right), \quad n=0, \cdots, M .
$$

${ }^{3}$ Band-limited chip waveforms would of course extend beyond the chip "boundaries" shown in Fig. 1. 
Consequently

$$
\begin{array}{r}
r(t)=A_{1} \tilde{c}_{1}(t)+\sum_{k=2}^{K_{\text {cff }}} A_{k}^{\prime} b_{k} \tilde{c}_{k}(t) e^{j \phi_{k}}+w(t), \\
t \in\left[0, M T_{s}\right] .
\end{array}
$$

The symbols of the interfering users are not of immediate interest, and they can be grouped with the amplitudes and phases as

$$
a_{(M+1)(k-2)+n+1}=A_{k} b_{k, n-2} e^{j \phi_{k}}, \quad n=1 \cdots M+1 .
$$

Furthermore, we use the vector notation $\boldsymbol{a}=\left[a_{2} \cdots a_{K_{\mathrm{cff}}}\right]^{\top}$. The first step toward deriving an acquisition algorithm is to convert the continuous signal model at the receiver into an equivalent discrete model. The generation of discrete system models for asynchronous CDMA systems has been considered in detail in [15]. It essentially involves the projection of $r(t)$ onto a set of basis functions $\left\{g_{n}(t)\right\}$, i.e.,

$$
\begin{aligned}
y_{n} & =\left\langle r(t), g_{n}(t)\right\rangle \\
& =\int_{0}^{T} r(t) g_{n}^{*}(t) d t \quad \text { for } n=1 \cdots D
\end{aligned}
$$

where $T$ is the observation interval $M T_{s}$, and $D$ could represent a dimensional restriction in the system. Since this is a linear operation, the resulting vector $\boldsymbol{y}=\left[y_{1}, y_{2}, \cdots, y_{D}\right]^{\top}$ can be represented as

$$
\boldsymbol{y}=a_{1} \boldsymbol{u}_{1}\left(\tau_{1}\right)+\boldsymbol{U} \boldsymbol{a}+\boldsymbol{w}
$$

where $u_{i j}=\left\langle g_{i}(t), \tilde{c}_{j}(t)\right\rangle$, and $\boldsymbol{U}=\left[\boldsymbol{u}_{2}, \cdots, u_{K_{\mathrm{cff}}}\right]$ is a matrix of only the interfering user vectors. The vector corresponding to the new user $u_{1}\left(\tau_{1}\right)$ is separated out and the dependence of $\boldsymbol{u}_{1}$ on $\tau_{1}$ is explicitly shown. Also, note that $a_{1}=A_{1}$ is always positive, and $\boldsymbol{w}$ is a complex circularly Gaussian (CCG) vector with $w_{i}=\left\langle g_{i}(t), w(t)\right\rangle$.

The basis functions we use in arriving at (6) correspond to chip-matched filtering with respect to an arbitrary timing reference (see Fig. 2), i.e., $g_{n}(t)=\psi\left(t-n T_{c}\right)$, for $n=0 \cdots M N-$ 1 , and hence $D=M N$. The acquisition problem we consider can then be phrased as follows:

Given $\boldsymbol{y}$, and the corresponding matrix $\boldsymbol{U}$, estimate $\tau_{1}$ without assuming knowledge of $\boldsymbol{a}$.

Note that $y \in \mathcal{C}^{M N}$ and the matrix $U$ is of size $M N \times(M+$ $1)(K-1)$. For large $K$ and $N$, processing $U$ would be computationally intensive. To overcome this problem, and to create another degree of freedom at the same time, we could choose to cancel only $K_{c}$ users out of the $K-1$ interfering users. So, in general, $\boldsymbol{U}$ would be of size $M N \times(M+1) K_{c}$. We model interference from the remaining $K_{r}=K-K_{c}-1$ users as white Gaussian noise and include it into $w$ to get $\boldsymbol{w}_{\boldsymbol{I}}$. Since we have an asynchronous setting, the sequence of each effective vector would in general be a colored sequence, but we make this modeling assumption for the purpose of simplification. Using t to denote the Hermitian of a matrix, the covariance matrix of $\boldsymbol{w}_{\boldsymbol{I}}$ is given by $\boldsymbol{R}_{\boldsymbol{w}_{\boldsymbol{I}}}=(1 / 2) E\left(\boldsymbol{w}_{\boldsymbol{I}} \boldsymbol{w}_{\boldsymbol{I}}^{\dagger}\right)=\sigma_{\boldsymbol{I}}^{2} \boldsymbol{I}_{M N}$, where [15]

$$
\sigma_{\boldsymbol{I}}^{2} \approx N_{0}+\sum_{k=K_{c}+2}^{K} \frac{\mathcal{E}_{k}}{N} \sigma_{\psi}^{2}
$$

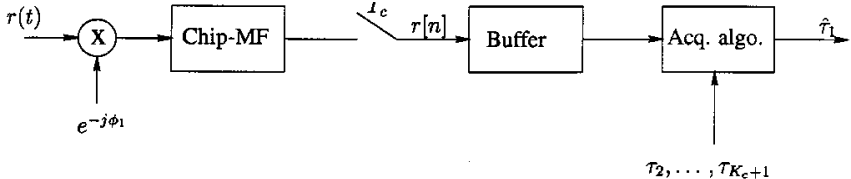

Fig. 2. Receiver front-end for coherent acquisition scheme with chip-matched filtering. For the noncoherent case, the initial rotation by $\phi_{1}$ would not be present.

and $\sigma_{\psi}^{2}=\int_{-\infty}^{\infty} R_{\psi}^{2}(\alpha) d \alpha$, with $R_{\psi}(\alpha)$ being the autocorrelation function of the chip waveform

$$
R_{\psi}(\alpha)=\int_{-\infty}^{\infty} \psi(t) \psi\left(t-\alpha T_{c}\right) d t
$$

More generally, $\boldsymbol{w}_{\boldsymbol{I}}$ could include out-of-cell interference as well.

Finally, we define the interference space $\mathcal{S}$ to be the column space of $\boldsymbol{U}$ and the noise space $\mathcal{N}$ to be its orthogonal component in $\mathcal{C}^{M N}$. This is a slight abuse of terminology since the "noise" space does contain a component of the new user's signal. The dimensions of $\mathcal{S}$ and $\mathcal{N}$ are

$$
d_{S}=(M+1) K_{c} \quad \text { and } \quad d_{N}=M N-(M+1) K_{c}
$$

respectively, so $M$ and $K_{c}$ are restricted to ensure $M N-(M+$ 1) $K_{c}>0$.

Remark 1: If the interfering signals are linearly dependent, the signal space dimension gets reduced. In general, we then have $d_{S} \leq(M+1) K_{c}$ and $d_{N} \geq M N-(M+1) K_{c}$, but the following analysis remains unchanged.

\section{MAXIMUM-LIKELIHOOD SCHEME}

Resolving $\boldsymbol{y}$ into its components in $\mathcal{S}$ and $\mathcal{N}, \boldsymbol{y}=\boldsymbol{y}^{\|}+\boldsymbol{y}^{\perp}$, we can rewrite (6) as

$$
\boldsymbol{y}=\boldsymbol{y}^{\perp}+\boldsymbol{U} \boldsymbol{a}^{\prime}
$$

for an appropriately chosen $\boldsymbol{a}^{\prime}$. We will show (see Theorem 1) that $\boldsymbol{y}^{\perp}$ is sufficient for ML estimation of $\tau_{1}$ based on $\boldsymbol{y}$.

Since we are implicitly assuming a 2-norm over the vector space $\mathcal{C}^{M N}$, we may write $\boldsymbol{y}^{\perp}=\boldsymbol{y}-\boldsymbol{U} \boldsymbol{a}_{L S}$, where $\boldsymbol{a}_{L S}$ is the least squares solution to $\boldsymbol{y}=\boldsymbol{U} \boldsymbol{a}$. Our scheme relies on the QR method to solve this least squares problem [16]. The first step is a Gram-Schmidt orthogonalization, $\boldsymbol{U}=\boldsymbol{Q} R$, with $\boldsymbol{Q}$ orthogonal and $\boldsymbol{R}$ upper triangular. Then, defining $\boldsymbol{B}=\boldsymbol{I}-$ $Q Q^{\dagger}$, we have

$$
\boldsymbol{y}^{\perp}=\left(\boldsymbol{I}-\boldsymbol{Q} Q^{\dagger}\right) \boldsymbol{y}=\boldsymbol{B} y=a_{1} \boldsymbol{B} u_{1}+\boldsymbol{B} w_{\boldsymbol{I}}
$$

The matrix $B$ is a projection matrix, which projects $\boldsymbol{y}$ into the noise space $\mathcal{N}$, and yields a vector independent of the powers of the $K_{c}$ users being cancelled. To ensure that $\boldsymbol{B} u_{1}$ in (9) is nonzero, we need to have $\boldsymbol{u}_{1}$ linearly independent of the interfering vectors. But, as stated in Remark 1, these interfering vectors need not be linearly independent among themselves. We have the following results.

Lemma 1: The matrix $\boldsymbol{B}$ satisfies the following properties.

i) $B^{\dagger}=B, B^{\dagger} B=B^{2}=B$.

ii) $\boldsymbol{B}$ has eigenvalues 0 (order $\left.d_{S}\right)$ and 1 (order $\left.d_{N}\right)$.

iii) $\operatorname{trace}(\boldsymbol{B})=d_{N} \geq M N-(M+1) K_{c}$. 
Theorem 1: The vector $\boldsymbol{y}^{\perp}$ is a sufficient statistic for the estimation of the delay, and the ML solution satisfies

$$
\left[\hat{\tau}_{1}, \hat{a}_{1}\right]=\arg \max _{\tau_{1}, a_{1}} p\left(\boldsymbol{y}^{\perp} \mid \tau_{1}, a_{1}\right) .
$$

Proof: Since knowledge of $\boldsymbol{a}$ is not assumed, the ML solution for $\tau_{1}$ is obtained by maximizing the likelihood function of $\boldsymbol{y}$ over $\boldsymbol{a}, a_{1}$ and $\tau_{1}$ in (6)

$$
\left[\hat{\tau}_{1}, \hat{a}_{1}\right]=\arg \max _{\tau_{1}, a_{1}} \max _{\boldsymbol{a}} p\left(\boldsymbol{y} \mid a, \tau_{1}, a_{1}\right)
$$

Let $\boldsymbol{K}=\boldsymbol{U}^{\dagger} \boldsymbol{U}$ be the covariance matrix of the interfering users, and assume, for now, that it is invertible. Then, for $\left[a_{1}, \tau_{1}\right]$ fixed, the maximization over $\boldsymbol{a}$ becomes

$$
\min _{\boldsymbol{a}}(\boldsymbol{z}-\boldsymbol{U} \boldsymbol{a})^{\dagger}(\boldsymbol{z}-\boldsymbol{U} \boldsymbol{a})=\max _{\boldsymbol{a}} 2 \boldsymbol{z}^{\dagger} \boldsymbol{U} \boldsymbol{a}-\boldsymbol{a}^{\dagger} \boldsymbol{K} \boldsymbol{a}
$$

where $\boldsymbol{z}=\boldsymbol{y}-a_{1} \boldsymbol{u}_{1}\left(\tau_{1}\right)$. It follows that the maximizing $\boldsymbol{a}$ in the above equation is, $\hat{\boldsymbol{a}}=\boldsymbol{K}^{-1} \boldsymbol{U}^{\dagger} \boldsymbol{z}$ and the corresponding metric is

$$
\left(\boldsymbol{z}-\boldsymbol{U} \boldsymbol{K}^{-1} \boldsymbol{U}^{\dagger} \boldsymbol{z}\right)^{\dagger}\left(\boldsymbol{z}-\boldsymbol{U} \boldsymbol{K}^{-1} \boldsymbol{U}^{\dagger} \boldsymbol{z}\right)
$$

Finally, noting that

$$
\boldsymbol{I}-\boldsymbol{U} \boldsymbol{K}^{-1} \boldsymbol{U}^{\dagger}=I-\boldsymbol{Q} \boldsymbol{Q}^{\dagger}=\boldsymbol{B}
$$

we have

$$
\begin{aligned}
{\left[\hat{\tau}_{1}, \hat{a}_{1}\right] } & =\arg \min _{a_{1}, \tau_{1}} \boldsymbol{z}^{\dagger} \boldsymbol{B} \boldsymbol{z} \\
& =\arg \min _{a_{1}, \tau_{1}}\left(\boldsymbol{y}-a_{1} \boldsymbol{u}_{1}\right)^{\dagger} \boldsymbol{B}\left(\boldsymbol{y}-a_{1} \boldsymbol{u}_{1}\right) \\
& =\arg \max _{a_{1}, \tau_{1}} 2 a_{1} \operatorname{Re}\left\{\boldsymbol{u}_{1}^{\dagger} \boldsymbol{B} \boldsymbol{y}\right\}-a_{1}^{2} \boldsymbol{u}_{1}^{\dagger} \boldsymbol{B} \boldsymbol{u}_{1}
\end{aligned}
$$

where we have used Lemma 1. From the above equation, it is clear that $\boldsymbol{y}^{\perp}=\boldsymbol{B} \boldsymbol{y}$ is sufficient for ML estimation of $\tau_{1}$ and that (10) is true.

If the interfering signals are linearly dependent, the steps given above for finding the ML solution may be modified by reducing $\boldsymbol{U}$ and $\boldsymbol{a}$ in (6) to an appropriate $\boldsymbol{U}^{\prime}$ and $\boldsymbol{a}^{\prime}$, such that $U^{\prime \dagger} U^{\prime}=\boldsymbol{K}^{\prime}$ is invertible. If we carry the steps through, it is easy to show that the solution for $\hat{\tau}_{1}$ given in (14) remains unchanged.

From (14), we have

$$
\begin{aligned}
\hat{a}_{1} & =\frac{\operatorname{Re}\left\{\boldsymbol{u}_{1}(\hat{\tau})^{\dagger} \boldsymbol{B} \boldsymbol{y}\right\}}{\boldsymbol{u}_{1}(\hat{\tau})^{\dagger} \boldsymbol{B} \boldsymbol{u}_{1}(\hat{\tau})} \\
\hat{\tau}_{1} & =\arg \max _{\tau: \hat{a}_{1}(\tau)>0} \frac{\left(\operatorname{Re}\left\{\boldsymbol{u}_{1}(\tau)^{\dagger} \boldsymbol{B} \boldsymbol{y}\right\}\right)^{2}}{\boldsymbol{u}_{1}(\tau)^{\dagger} \boldsymbol{B} \boldsymbol{u}_{1}(\tau)} \\
& =\arg \max _{\tau} \frac{\operatorname{Re}\left\{\boldsymbol{u}_{1}(\tau)^{\dagger} \boldsymbol{B} \boldsymbol{y}\right\}}{\sqrt{\boldsymbol{u}_{1}(\tau)^{\dagger} \boldsymbol{B} \boldsymbol{u}_{1}(\tau)}} \\
& =\arg \max _{\tau} \frac{\operatorname{Re}\left\{\boldsymbol{u}_{1}(\tau)^{\dagger} \boldsymbol{B} \boldsymbol{y}\right\}}{\left\|\boldsymbol{B} u_{1}(\tau)\right\|}=f_{\mathrm{ML}}(\tau)
\end{aligned}
$$

where (16) uses the restriction $a_{1}>0$. The above derivation for $f_{\mathrm{ML}}(\tau)$ assumes that $\left\|\boldsymbol{B} u_{1}(\tau)\right\| \neq 0$, i.e., $\boldsymbol{u}_{1}(\tau)$ does not belong entirely to the interference space $\mathcal{S}$. If $\boldsymbol{u}_{1}(\tau)$ is contained in $\mathcal{S}, y^{\perp}$ contains no information about $\tau_{1}$, but this happens with probability zero if $d_{N}>0$.

We still need to maximize the statistic in (17) over $\tau$ to get an estimate of the delay. This maximization can be done analytically when the chip waveform is time-limited to $\left[0, T_{c}\right]$. In this case, since $u_{i k}=\left\langle\psi\left(t-i T_{c}\right), \tilde{c}_{k}(t)\right\rangle$, we have

$$
\boldsymbol{u}_{k}\left(\tau_{k}\right)=R_{\psi}\left(\alpha_{k}\right) \tilde{\boldsymbol{c}}_{k}\left(n_{k}\right)+R_{\psi}\left(1-\alpha_{k}\right) \tilde{\boldsymbol{c}}_{k}\left(n_{k}+1\right)
$$

where $\tau_{k}=\left(n_{k}+\alpha_{k}\right) T_{c}$, and $\tilde{c}_{k}\left(n_{k}\right)$ is the vector corresponding to the sequence of $k$ th effective user. The same applies for $\boldsymbol{u}_{1}(\tau)$ with $\tau=(n+\alpha) T_{c}$ ranging over the uncertainty interval $\left[0, L T_{c}\right]$. The optimization (17) can then be done in a manner similar to that in the literature (see, e.g., [4] and [3]). However, it is important to note that this analytical maximization strategy does not carry over to the realistic case where we have band-limited chip waveforms. Furthermore, the solution for (7) with chip waveforms time-limited to $\left[0, T_{c}\right]$ results in poor performance when applied to the case with band-limited chip waveforms. Thus, analysis with such time-limited waveforms may be of little value in designing acquisition schemes for practical systems [17]. In our study of the acquisition problem, we use a sinc pulse truncated to 9 chips and perform a brute force maximization over the one-dimensional parameter $\tau$. This consists of generating finely sampled versions of the users' signals and evaluating the statistic at successive delays $\tau$ separated by the sample spacing. Numerical simulations show that the resolution can be kept reasonably low without significant degradation in performance.

\section{EXTENSIONS OF THE ML SCHEME}

\section{A. Noncoherent Acquisition}

Since we have assumed a model with linear modulation, noncoherent detection of the symbols of the users is not possible. However, it is still possible to do the acquisition in a noncoherent manner. This is also necessitated in cases where the carrier phase cannot be acquired before the sequence delay. After the spreading sequence is acquired and tracked, the carrier phase can be tracked using a phase-locked loop. In this context, it must be noted that while the IS-95 design [10] uses 64-ary orthogonal modulation and noncoherent detection on the reverse link, future standards [11] may employ only the $Q$ channel for user data, with a pilot channel provided on the $I$ channel to help in initial acquisition.

The ML acquisition scheme derived in the previous section is easily modified for the noncoherent case. We assume that the phase remains constant over the observation interval of duration $T=M T_{s}$. This assumption is valid so long as $\Delta f_{c} T \ll 1$, where $\Delta f_{c}$ is the frequency mismatch between the transmitter and the receiver carriers. Following similar discretization steps as in the coherent case, we have

$$
\boldsymbol{y}=a_{1} \boldsymbol{u}_{1}\left(\tau_{1}\right) e^{j \phi_{1}}+\boldsymbol{U} \boldsymbol{a}+\boldsymbol{w}
$$

where $\phi_{1}$ is the unknown phase and $\boldsymbol{a}$ is defined analogous to (5). To derive the ML delay estimator, we simply include $\phi_{1}$ into the arguments over which the likelihood function of $\boldsymbol{y}$ is 
maximized. It is easily verified that $\boldsymbol{y}^{\perp}=\boldsymbol{B y}$ form sufficient statistics in this case as well. Consequently, we have

$$
\left[\hat{\tau}_{1}, \hat{a}_{1}, \hat{\phi}_{1}\right]=\arg \max _{\tau_{1}, a_{1}, \phi_{1}} p\left(\boldsymbol{y}^{\perp} \mid \tau_{1}, a_{1}, \phi_{1}\right)
$$

Using Lemma 1 again, the statistic to be maximized is

$$
2 a_{1} \operatorname{Re}\left\{\boldsymbol{u}_{1}^{\dagger} \boldsymbol{B} \boldsymbol{y} e^{j \phi_{1}}\right\}-a_{1}^{2} \boldsymbol{u}_{1}^{\top} \boldsymbol{B} \boldsymbol{u}_{1}
$$

After maximizing over $\phi_{1}$, the statistic reduces to

$$
2 a_{1}\left|\boldsymbol{u}_{1}^{\dagger} \boldsymbol{B} \boldsymbol{y}\right|-a_{1}^{2} \boldsymbol{u}_{1}^{\top} \boldsymbol{B} \boldsymbol{u}_{1}
$$

which is similar to the metric in (14). Consequently, the ML delay estimator is given by

$$
\hat{\tau}_{1}=\arg \max _{\tau} \frac{\left|\boldsymbol{u}_{1}(\tau)^{\top} \boldsymbol{B} \boldsymbol{y}\right|^{2}}{u_{1}(\tau)^{\top} \boldsymbol{B} \boldsymbol{u}_{1}(\tau)} .
$$

\section{B. Acquisition with Multiple Windows}

The acquisition strategy thus far involves processing a single window of observations consisting of $M N$ chips. The dimension of the "noise" space increases with $M$, and hence the acquisition scheme may be expected to perform better as $M$ increases. However, the size of the matrices involved grows linearly with $M$, making the processing computationally intensive for large values of $M$. Specifically, consider the case where the observation is over $b M N$ chips. Instead of using the ML scheme over the window length of $b M N$ for $\boldsymbol{y}$, a suboptimal but computationally less intensive approach would be to repeat the processing (9) for each block and to combine the results appropriately. For the coherent case, this can be done as follows.

Given $\boldsymbol{y}_{i}^{\perp}=\boldsymbol{B}_{i} \boldsymbol{y}_{i}, i=1, \cdots, b$, we could again pose an ML problem as in (10). Defining $\boldsymbol{y}_{\text {eff }}^{\perp}=\left[\left(\boldsymbol{y}_{1}^{\perp}\right)^{\top} \cdots\left(\boldsymbol{y}_{b}^{\perp}\right)^{\top}\right]^{\top}$, we need to maximize $p\left(\boldsymbol{y}_{\text {eff }}^{\perp} \mid a_{1}, \tau_{1}\right)$. But $\boldsymbol{y}_{\text {eff }}^{\perp}=\boldsymbol{B}_{\text {eff }} \boldsymbol{y}$ where $\boldsymbol{y}$ is a concatenation of the $\left\{\boldsymbol{y}_{\boldsymbol{i}}\right\}$ vectors, and $\boldsymbol{B}_{\text {eff }}=\operatorname{diag}\left(\boldsymbol{B}_{\boldsymbol{i}}\right)$. Hence, the solution to (10) applies, with $\boldsymbol{B}$ replaced by $\boldsymbol{B}_{\text {eff }}$

$$
\begin{aligned}
\hat{\tau}_{1} & =\arg \max _{\tau} \frac{\operatorname{Re}\left\{\boldsymbol{u}_{1}(\tau)^{\dagger} \boldsymbol{B}_{\mathrm{eff}} \boldsymbol{y}\right\}}{\left\|\boldsymbol{B}_{\mathrm{eff}} \boldsymbol{u}_{1}(\tau)\right\|} \\
& =\arg \max _{\tau} \frac{\sum_{i=1}^{b} \operatorname{Re}\left\{\boldsymbol{u}_{1, i}(\tau)^{\dagger} \boldsymbol{B}_{i} \boldsymbol{y}_{i}\right\}}{\sqrt{\sum_{i=1}^{b} \boldsymbol{u}_{1, i}(\tau)^{\dagger} \boldsymbol{B}_{i} \boldsymbol{u}_{1, i}(\tau)}} .
\end{aligned}
$$

When this suboptimal approach is used, note that the restriction $K_{c}$ is $M N-(M+1) K_{c}>0$, which is more stringent compared to the condition $b M N-(b M+1) K_{c}>0$ for the optimal approach. It is also of interest to note that for a system with short sequences, there is further reduction in the computational complexity, since $B$ does not change with the window index $i$. However, this may not be true in a general setting for short sequences if the delays of the interfering users change during the observation period. Also, with short sequences, it may happen that $\boldsymbol{u}_{1}\left(\tau_{1}\right)$ has only a small component in the noise space $\mathcal{N}$ for a particular realization of the delays of the interfering users. Using long sequences overcomes this problem since the sequences change over each window.

Finally, following (21), the above block processing approach is easily modified for the noncoherent case. In this case, we have the additional advantage that the unknown carrier phase can be ensured to remain approximately constant over each window by choosing $M$ sufficiently small.

\section{Approximation to the $M L$ Scheme}

Consider again our basic statistic in (14). It contains the term $a_{1}^{2} \boldsymbol{u}_{1}\left(\tau_{1}\right)^{\dagger} \boldsymbol{B} \boldsymbol{u}_{1}\left(\tau_{1}\right)$. An approximation to the ML solution may be obtained by ignoring the variation of this term with $\tau_{1}$, i.e., by assuming that the norm of the projection of $u_{1}$ into the noise space is constant. Then the ML estimate gets modified as

$$
\hat{\tau}_{1}=\arg \max _{\tau} \operatorname{Re}\left\{\boldsymbol{u}_{1}(\tau)^{\dagger} \boldsymbol{B} \boldsymbol{y}\right\}=f_{\mathrm{AML}}(\tau) .
$$

We refer to this as the AML (approximate maximum-likelihood) solution. Comparing to (9), we see that AML solution essentially amounts to ignoring the coloring of the noise and applying a filter matched to $\boldsymbol{B} \boldsymbol{u}_{1}$ to $\boldsymbol{y}^{\perp}$.

\section{ACQUISITION PERFORMANCE}

The ML acquisition scheme derived involves collecting chip-matched filter outputs from one or more windows and processing them to get an estimate of the delay. The acquisition time thus depends on the length and number of windows used as well as the computational resources at the receiver, and is hence difficult to characterize in general. The acquisition stage is usually followed by a tracking device, and it seems reasonable to consider instead performance in terms of probability of acquisition, defined as

$$
P_{\mathrm{ACQ}}=\operatorname{Prob}\left\{\left|\hat{\tau}_{1}-\tau_{1}\right|<\delta T_{c}\right\}
$$

where it is assumed that the tracking device requires an initial accuracy of $\delta T_{c}$. Correspondingly, the probability of acquisition error is $P_{e}=1-P_{\mathrm{ACQ}}$.

Ideally, we would like to get an analytical handle on $P_{e}$ for the ML scheme, but this seems to be a difficult problem. As a first step in the simplification, we consider the AML scheme (23) instead. We also assume that the chip boundaries of the new user are known a priori. This means that the fractional delay of the new user $\alpha_{1}$ is known, and hence the delay estimation problem reduces to that of detecting just the integral delay $n_{1}$, which can take on values in $\{0, \cdots, L-1\}$. We further assume that the chip-MF output samples are in fact aligned with respect to the new user, so that $\alpha_{1}=0 .{ }^{4}$ However, it must be noted that the interfering users are allowed to be completely asynchronous, so we are not considering a chip-synchronous situation. The resulting analysis could provide an insight into the effect of the various parameters involved in the system and allow for a comparison to the conventional acquisition scheme. Also, it will be

\footnotetext{
${ }^{4}$ For signals (approximately) band-limited to $W=1 / 2 T_{c}$, such as the ones we consider in this paper, assuming $\alpha_{1}=0$ would lead to no loss in generality.
} 
of interest to compare the results obtained to the performance in the case where the chip boundaries are not known.

\section{A. Analysis with Known Chip Boundaries}

When the chip boundaries of the new user are known and $\alpha_{1}=0$, the delay estimation problem reduces to a detection problem. The AML solution for the detection problem is

$$
\hat{\tau}_{1}=\arg \max _{n \in\{0 \cdots L-1\}} \tilde{\boldsymbol{c}}_{1}(n)^{\dagger} B \boldsymbol{y}
$$

where $u_{1}(\tau)=\tilde{\boldsymbol{c}}_{1}(n)$. We model $\tilde{\boldsymbol{c}}_{1}(n)$ as an i.i.d. random vector of length $M N$, with each element equally likely to take on the values $\{\exp (j 2 \pi k / P)\}_{k=0}^{P-1}$ on the unit circle. Clearly, $P=2$ corresponds to binary sequences and $P=4$ corresponds to quadri-phase sequences (employed in [11]). Using (9) and (25), the AML statistic under the hypothesis $\tau=n$ is

$$
X_{n}=a_{1} \tilde{\boldsymbol{c}}_{1}(n)^{\dagger} \boldsymbol{B} \tilde{\boldsymbol{c}}_{1}\left(n_{1}\right)+\tilde{\boldsymbol{c}}_{1}(n)^{\dagger} \boldsymbol{B} \boldsymbol{w}_{\boldsymbol{I}}
$$

We assume that $a_{1}$ is a fixed nonrandom parameter. For a given noise level $N_{0}$, this corresponds to assuming that we are operating at a fixed SNR $=\mathcal{E}_{1} / N_{0}$. The matrix $\boldsymbol{B}$ is a function of the spreading sequences and delays of the users being cancelled, and may be modeled as a random matrix. Further, we model $X_{n}$ as a circularly complex Gaussian random variable, ${ }^{5}$ where the randomness comes from $\boldsymbol{w}_{\boldsymbol{I}}, \tilde{\boldsymbol{c}}_{1}$, and $\boldsymbol{B}$. Hence, the distribution of $X_{n}$ is completely defined by the first two moments. We assume that $\tilde{c}_{1, i}(n)$, the $i$ th chip of $\tilde{c}_{1}(n)$, is independent of $c_{1, j}\left(n^{\prime}\right)$ unless $i=j$ and $n=n^{\prime}$. This is certainly not true across delays [e.g., $\left.c_{1, i}(n)=c_{1, i+1}(n-1)\right]$, but the statistics obtained involve averaging over $\boldsymbol{B}$ as well, and the above independence assumption is a good approximation. Under these assumptions, it is shown in the Appendix that

$$
\begin{aligned}
& E\left[X_{n}\right]= \begin{cases}0, & \text { if } n \neq n_{1} \\
m_{d}=a_{1} d_{N}, & \text { if } n=n_{1}\end{cases} \\
& \frac{1}{2} \operatorname{Var}\left[X_{n}\right]= \begin{cases}\sigma_{f}^{2}=\left(\frac{a_{1}^{2}}{2}+\sigma_{\boldsymbol{I}}^{2}\right) d_{N}, & \text { if } n \neq n_{1} \\
\sigma_{d}^{2} \leq\left(\frac{a_{1}^{2} d_{S}}{M N}+\sigma_{\boldsymbol{I}}^{2}\right) d_{N}, & \text { if } n=n_{1}\end{cases} \\
& E\left[X_{n} X_{n^{\prime}}\right]=0, \quad \text { for } n \neq n^{\prime} \text {. }
\end{aligned}
$$

Here, the variance of the complex random variable $X_{n}$, $\operatorname{Var}\left[X_{n}\right]=E\left|X_{n}-E X_{n}\right|^{2}$. We note that, for the particular case of $P=2$, we have real sequences and the the expressions for $\sigma_{f}^{2}$ and $\sigma_{d}^{2}$ have $a_{1}^{2}$ replaced by $2 a_{1}^{2}$. We also note from the Appendix that the bound on $\sigma_{d}^{2}$ is based on the more general result

$$
\frac{\operatorname{Var}\left(Q_{n}\right)}{\left(E Q_{n}\right)^{2}} \leq \frac{d_{S}}{d_{N} M N}
$$

where $Q_{n}=\tilde{\boldsymbol{c}}_{1}(n)^{\top} \boldsymbol{B} \tilde{\boldsymbol{c}}_{1}(n)$.

Now, the AML statistic was derived by assuming that the denominator of the ML statistic $q_{n}$ was independent of $n$. Hence,

\footnotetext{
${ }^{5}$ For the detection problem, the standard Gaussian approximation is known to be unreliable at low error probabilities [18]. But the error probability requirements are typically far less stringent on the acquisition stage and the Gaussian approximation could prove useful.
}

assuming known chip boundaries and that the above Gaussian approximations are reasonably accurate, the performance results for the ML scheme may be expected to come close to those for the AML scheme when the bound (28) is small. This happens when $d_{S} \ll M N$, i.e., when we are operating far away from the dimensional limit, ${ }^{6}$ i.e, when the number of users cancelled $K_{c}$ is kept small in comparison to $N$. Since reduction in computational complexity may motivate the choice of a low value for $K_{c}$, this regime of operation is of practical interest.

Continuing with the analysis, we have that, with the Gaussian approximation, $X_{n}$ are i.i.d. $\mathcal{C N}\left(0, \sigma_{f}^{2}\right)$ for $n \neq n_{1}$, and $X_{n_{1}} \sim \mathcal{C N}\left(m_{d}, \sigma_{d}^{2}\right)$ independent of the other $X_{n}$. Here, $X \sim \mathcal{C N}\left(\mu, \sigma^{2}\right)$ denotes the CCG distribution with mean $E X=\mu$ and variance $E|X-\mu|^{2}=2 \sigma^{2}$. Hence the probability of acquisition, $P_{\mathrm{ACQ}}$, is given by

$$
\begin{aligned}
& P_{\mathrm{ACQ}}= P\left\{\operatorname{Re}\left\{X_{n_{1}}\right\}>\max _{n \neq n_{1}} \operatorname{Re}\left\{X_{n}\right\}\right\} \\
&= \frac{1}{\sigma_{d} \sqrt{2 \pi}} \int_{-\infty}^{\infty} e^{-\left(\left(x-m_{d}\right)^{2} / 2 \sigma_{d}^{2}\right)} \\
& \cdot\left(1-Q\left(\frac{x}{\sigma_{f}}\right)\right)^{L-1} d x .
\end{aligned}
$$

Note that (27) gives only an upper bound on $\sigma_{d}$ based on our approximate model, and the $P_{e}$ obtained using this bound could overestimate the actual $P_{e}$. Since the AML scheme is based on assuming $Q_{n}$ is a constant, a heuristic approximation can be obtained by setting $Q_{n_{1}}=E\left(Q_{n_{1}}\right)=d_{N}$. We then have

$$
\sigma_{d}^{2} \approx \sigma_{\boldsymbol{I}}^{2} d_{N}
$$

and the gap between this approximation and the bound gets reduced when $a_{1}^{2} d_{S} / M N \ll \sigma_{I}^{2}$ or, equivalently, when the effective SNR $\ll M N / d_{S}$. This is again the regime of small $K_{c}$, since the remaining interferers would also contribute to lowering the effective SNR.

For the noncoherent case, the AML statistic would be $\left|X_{n}\right|$ that follows the standard Rayleigh and Ricean distributions:

$$
p_{\left|X_{n}\right|}(x)= \begin{cases}\frac{x}{\sigma_{f}^{2}} e^{-\left(x^{2} / 2 \sigma_{f}^{2}\right)}, & \text { if } n \neq n_{1} \\ \frac{x}{\sigma_{d}^{2}} I_{0}\left(\frac{x m_{d}}{\sigma_{d}^{2}}\right) e^{-\left(\left(x^{2}+m_{d}^{2}\right) / 2 \sigma_{d}^{2}\right)}, & \text { if } n=n_{1}\end{cases}
$$

where $I_{0}(\cdot)$ is the zeroth modified Bessel function of the first kind. Noting that the cdf of the Rayleigh random variable above is

$$
F_{\left|X_{n}\right|}(y)=\int_{0}^{y} \frac{x}{\sigma_{f}^{2}} e^{-\left(x^{2} / 2 \sigma_{f}^{2}\right)} d x=1-e^{-\left(y^{2} / 2 \sigma_{f}^{2}\right)}
$$

the acquisition probability is given by

$$
\begin{aligned}
P_{\mathrm{ACQ}}= & \int_{0}^{\infty} \frac{y}{\sigma_{d}^{2}} I_{0}\left(\frac{y m_{d}}{\sigma_{d}^{2}}\right) e^{-\left(\left(y^{2}+m_{d}^{2}\right) / 2 \sigma_{d}^{2}\right)} \\
& \cdot\left(1-e^{-\left(y^{2} / 2 \sigma_{f}^{2}\right)}\right)^{L-1} d y .
\end{aligned}
$$

${ }^{6}$ The AML and ML detection schemes are obviously equivalent in the extreme case of $d_{S}=0$, which corresponds to single user acquisition. 
Thus far, the AML statistic analyzed is for a single block of chip-matched filter outputs. Analogous to the procedure (22), we can improve reliability by using multiple blocks. The AML estimator with multiple blocks is obtained by simply averaging over $b$ blocks to get

$$
X(\tau)=\frac{1}{b} \sum_{i=0}^{b-1} \boldsymbol{u}_{1, i}(\tau)^{\dagger} \boldsymbol{B}_{i} \boldsymbol{y}_{i} .
$$

The statistic is then given by $\operatorname{Re}\{X(\tau)\}$ for the coherent case, and by $|X(\tau)|$ for the noncoherent case. In the analysis, this averaging merely reduces $\sigma_{f}$ and $\sigma_{d}$ by a factor of $\sqrt{b}$.

\section{B. Numerical Results and Discussion}

We now study the performance of the ML and AML schemes through simulations. The schemes are compared to the conventional scheme (corresponding to $K_{c}=0$ ), and the fidelity of the approximate analysis is tested. Throughout the simulations we fix the processing gain at $N=31$ and the delay uncertainty ${ }^{7}$ at $L=31$. The chip waveform is taken to be a sinc pulse truncated to a length of 9 chips, and the chip sequences are assumed to be complex, taking on equally likely quadri-phase values $(P=4)$. The schemes allow for the choice of several design and specification parameters:

- $M$, the length of each block;

- $K_{c}$, the number of users cancelled;

- $b$, the number of blocks;

- $K$, the number of users in the cell;

- and the SNR of the new user, where SNR $=E_{1} / N_{0}$.

We also have the restriction that $M N-(M+1) K_{c}>0$. As $K_{c}$ approaches $N$, we will need $\operatorname{larger} M$, so $K_{c}$ will be limited by the allowable complexity. In all cases, we will assume that the users have equal received powers. If the powers are unequal, the improvement in performance depends on which of the users are cancelled. Evidently, for the same total power in the interferers, the improvement will be greater if the high powers users can be identified and cancelled. Instead of accurate power estimation, a crude metric from the demodulators of the interfering users might suffice to this end.

We begin with a case where chip boundaries are known and we are operating close to the dimensional limit: $M=1$ (so the dimensional limit is 15 interferers) and $K_{c}=13$. The total number of users in the system is taken to be $K=14$, and the statistics are averaged over $b=3$ windows [see (22) and (33)]. The variation of $P_{e}$ with SNR is shown in Fig. 3 for the ML and AML schemes. The use of the AML scheme leads to a loss of about $2.5 \mathrm{~dB}$ in the effective SNR at $P_{e}=0.1$. In addition, the AML scheme exhibits an error floor: $P_{e}$ does not go to zero as SNR $\rightarrow \infty$. But this floor can be lowered by averaging over a larger number of windows. The bound obtained using (27) is found to be quite loose, especially at higher values of the SNR, while the approximation (30) is only marginally good since we are close to the dimensional limit.

The same set of curves is shown in Fig. 4 for the case where we have $M=3$ and $b=1$ instead. The number of users cancelled is still kept at $K_{c}=13$, and hence $d_{S} / M N$ is small.

${ }^{7}$ Of course, in the case of long sequences it is not necessary that $L=N$.

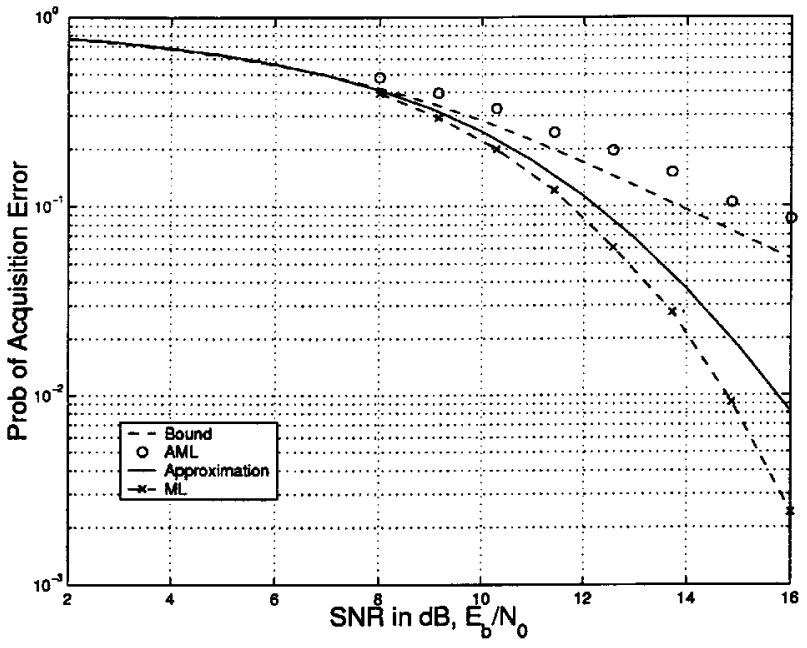

Fig. 3. Performance with variation in SNR, $M=1, b=3, N=31, L=31$, $K=14$.

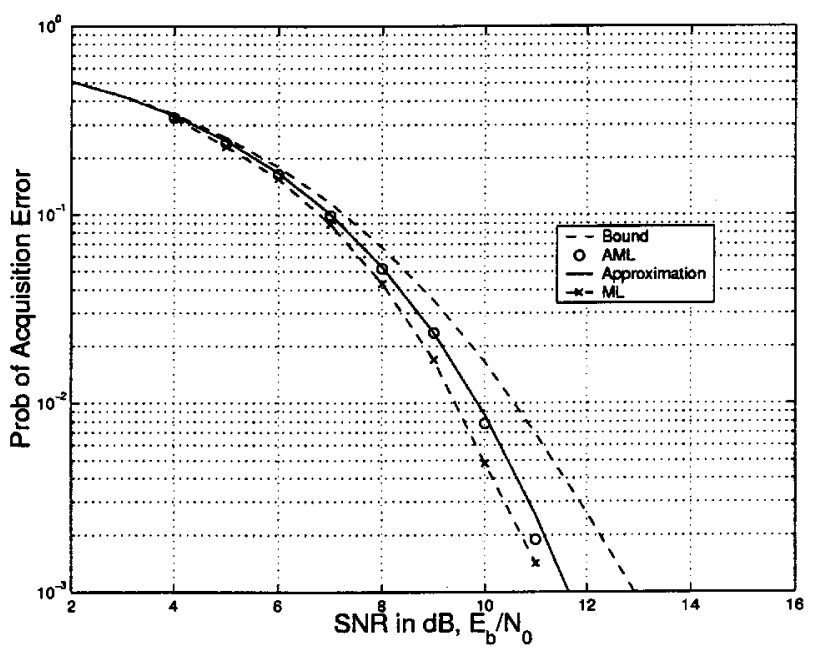

Fig. 4. Performance with variation in SNR. $M=3, b=1$.

The Gaussian approximation turns out to be quite good in this scenario, and the difference between the AML and ML schemes drops to less than $0.5 \mathrm{~dB}$ for the same $P_{e}=0.1$. Note also that the overall performance in this case is significantly better than the case where we had $M=1$ and $b=3$. Hence, for the same set of observations available, it is much better to process them as one window than multiple windows. But this comes at the expense of additional complexity. The bulk of the computation is taken up by the $O\left(b M^{3} N K_{c}^{2}\right)$ QR factorization, which varies as $M^{3}$ but is only linear in $b$.

In Fig. 5, we study the variation of $P_{e}$ with SNR for different values of $K_{c}$ with $M$ fixed at 3, along with the corresponding simulation results. The conventional parallel acquisition scheme corresponds to $K_{c}=0$, in which case $B=\boldsymbol{I}$. When $K_{c} \neq K-1$, the curves exhibit an interference floor. Note that, in spite of approximating the analysis by modeling the remaining interferers as white Gaussian, the analytical results obtained match quite well with the simulations. It is also of interest to note that, when the SNR is low, the decorrelating scheme $\left(K_{c}>0\right)$ actually performs worse than the conventional 


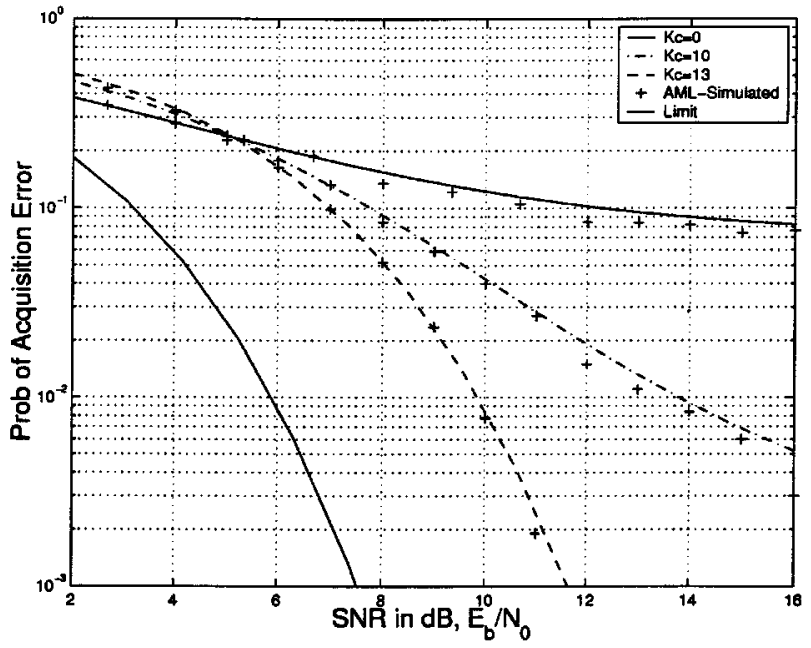

Fig. 5. Performance with variation in SNR and $K_{c}$.

scheme. This can be understood intuitively as follows. As $K_{c}$ increases, the variance of the residual interference and noise goes down. At the same time, the loss of the new user's component in the interference subspace increases. When the SNR is low, the latter effect is more significant. More rigorously, note from (27) and (30) that

$$
\sigma_{1}^{2}=\sigma_{f}^{2} \approx \sigma_{d}^{2} \approx \sigma_{I}^{2} d_{N}
$$

at low SNR. It then follows from (29) that $P_{e}$ a function of only $m_{d} / \sigma_{1}$, and is monotonically decreasing in $m_{d} / \sigma_{1}$. Furthermore, when all the users have equal powers, (7) gives

$$
\sigma_{\boldsymbol{I}}^{2}=N_{0}+\frac{\mathcal{E}}{N}\left(K-K_{c}-1\right) \sigma_{\psi}^{2}
$$

which implies that

$$
\frac{m_{d}}{\sigma_{1}}=\frac{a_{1} d_{N}}{\sigma_{I} \sqrt{d_{N}}}=\sqrt{\frac{2 \mathcal{E}\left(M N-(M+1) K_{c}\right)}{N N_{0}+\mathcal{E}(K-K c-1)}}
$$

since $\sigma_{\psi}^{2}=1$ for the sinc chip waveform. We can then easily show that $m_{d} / \sigma_{1}$ increases with $K_{c}$ (and hence, $P_{e}$ decreases with increasing $K_{c}$ ) when the SNR is above a threshold, i.e.,

$$
\frac{\mathcal{E}}{N_{0}}>\left(\frac{M}{M+1}-\frac{K-1}{N}\right)^{-1} \text {. }
$$

Clearly, when the SNR is below the threshold, $P_{e}$ increases with increasing $K_{c}$, meaning that the decorrelating scheme performs worse than the conventional scheme $\left(K_{c}=0\right)$ in this case. Note that this threshold behavior is independent of $b$, i.e., it is independent of any averaging done to improve the error probability. Hence, it is important to take the threshold SNR into account when designing the decorrelating scheme. We also note that similar behavior has been observed for the detection problem in [19], where the decorrelating detector is found to perform worse than the matched filter detector below a threshold SNR which depends on the loading factor $K / N$.

Returning to Fig. 5, for the parameters chosen, the threshold SNR computed using (35) is $4.8 \mathrm{~dB}$. This number matches well

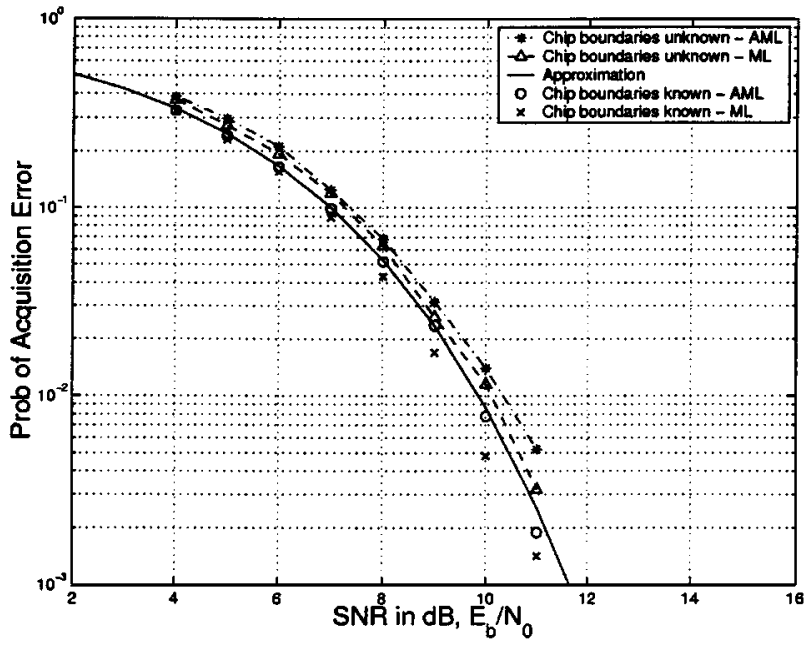

Fig. 6. Comparison with the case where chip boundaries are not known.

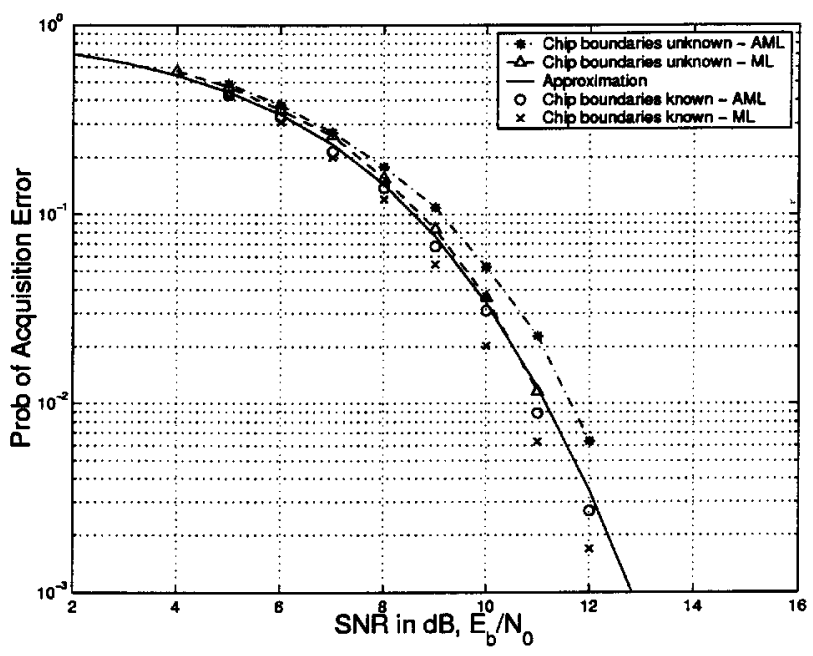

Fig. 7. Performance for the noncoherent case. $M=3, b=1$.

with the threshold seen in Fig. 5. The curves also show that significant gains over the conventional scheme are possible at high SNR's and high $K_{c}$. But these results assume equal powers for the interferers-the threshold SNR would be smaller and the gains could be significant even for small $K_{c}$ when the powers are unequal. Also, the best possible scenario is when there is perfect interference cancellation with the new user's signal remaining unchanged (e.g., when the users are perfectly orthogonal). This is equivalent to a single-user system at the same SNR and the corresponding $P_{e}$ curve is also shown as a limiting case.

Thus far, the numerical results assumed known chip boundaries. When the chip boundaries are unknown, we expect that the results will not be significantly affected [15]. This is confirmed by the results shown in Fig. 6, where the performance of the ML and AML schemes are shown with and without known chip boundaries. When the chip boundaries are unknown, the value of $\delta$ in the definition (24) of $P_{\mathrm{ACQ}}$ is set to 0.5 to allow for a fair comparison, and the resolution is set to $0.2 T_{c}$. It was found that a higher resolution does not significantly improve performance. Note that the interfering users are allowed to be completely asynchronous in all the cases. The same set of curves is shown in Fig. 7 for the noncoherent acquisition algorithm, 
which uses the maximization in (21), and the simulated performance is compared to the analytical approximation of (32). Note that the lack of phase information leads to a loss of about $1 \mathrm{~dB}$ at $P_{e}=0.01$.

The above results also indicate that the approximation resulting from the assumption of known chip boundaries is representative of performance in the actual band-limited system without this assumption. In particular, the observations made above regarding the effect of the parameters involved will hold for the actual system as well.

\section{CONCLUSION}

A maximum-likelihood delay estimation scheme applicable to DS/CDMA systems with long sequences was proposed and investigated. The scheme is closely related to the decorrelating detector and relies on the projection of the received vector into a changing but known "noise" space. This is similar to the projection used in the MUSIC algorithm [20] with the difference that the noise space here contains a component of the signal of interest. Along with several modifications of the ML estimator, a suboptimum approximate maximum-likelihood technique was presented and its performance analyzed under the assumption that the chip boundaries of the new user are known a priori. The analysis was based on Gaussian approximations and was found to give reliable results when the number of users cancelled is small compared to the processing gain. The performance of an actual band-limited system with unknown chip boundaries was found to match quite well with the analysis. It was seen that significant gains over the conventional scheme are possible at the expense of additional knowledge and computational complexity, so long as the operating SNR is above a precomputable threshold.

It is to be noted that the schemes derived in this paper rely on knowledge of the delays of the interfering users being cancelled. Results in [21] suggest that the ML scheme is reasonably robust to small errors in these delays, but this needs to be investigated further.

Extensions of the scheme to more general situations need to be investigated further. In particular, extension to frequency-selective fading channels is possible if we make the additional assumption that the channel estimates of the interfering users are known. The spreading vectors corresponding to the different taps of a single user can then be combined into a single vector and the above ML schemes can be applied. Research on this problem is currently underway.

\section{APPENDIX}

\section{DERIVATION OF STATISTICS (27)}

In this appendix, the results given in (27) are derived. We begin with (26), i.e.,

$$
X_{n}=a_{1} \tilde{\boldsymbol{c}}_{1}(n)^{\dagger} \boldsymbol{B} \tilde{\boldsymbol{c}}_{1}\left(n_{1}\right)+\tilde{\boldsymbol{c}}_{1}(n)^{\dagger} \boldsymbol{B} \boldsymbol{w}_{\boldsymbol{I}}
$$

For the sake of convenience, we use $c_{n}$ instead of $\tilde{c}_{1}(n)$ to denote the spreading vector of the new user corresponding to the delay hypothesis $\tau_{1}=n$. Also, let $c_{0}$ denote the vector corresponding to the correct delay, i.e., $\boldsymbol{c}_{0}=\tilde{\boldsymbol{c}}_{1}\left(n_{1}\right)$. Then, we have

$$
X_{n}=a_{1} c_{n}^{\dagger} B \boldsymbol{c}_{0}+\boldsymbol{c}_{n}^{\dagger} \boldsymbol{B} \boldsymbol{w}_{\boldsymbol{I}}
$$

\section{A. False Alarm Statistics}

In this case, the delay hypothesis $\tau=n$ is not equal to $n_{1}$. We model the sequences as i.i.d. random, and model $\boldsymbol{c}_{n}$ and $\boldsymbol{c}_{0}$ as uncorrelated random vectors for $n \neq n_{1}$. Clearly, this model does not hold when $\left|n-n_{1}\right|$ is less than the window length $M N$, since this would lead to an overlap between $\boldsymbol{c}_{n}$ and $\boldsymbol{c}_{0}$. But we make this heuristic assumption to simplify the analysis. It follows that

$$
\begin{aligned}
E\left(X_{n}\right) & =a_{1} E\left(c_{n}^{\dagger} \boldsymbol{B} \boldsymbol{c}_{0}\right)+E\left(\boldsymbol{c}_{n}^{\dagger} \boldsymbol{B} w_{\boldsymbol{I}}\right) \\
& \stackrel{(a)}{=} a_{1} E\left(\boldsymbol{c}_{n}^{\dagger} \boldsymbol{B} \boldsymbol{c}_{0}\right) \\
& =a_{1} E\left(\sum_{i, j} b_{i j} c_{n, i} c_{0, j}\right) \stackrel{(b)}{=} 0
\end{aligned}
$$

where (a) follows since the noise is zero mean and independent of the signal, and (b) can be seen by simply conditioning on $\boldsymbol{B}$ first. Note that $a_{1}$ is assumed to be a fixed nonrandom parameter. The second order statistics can be found as

$$
\begin{aligned}
\sigma_{f}^{2} & =\frac{1}{2} \operatorname{Var}\left(X_{n}\right)=\frac{1}{2} E\left(X_{n} X_{n}^{\star}\right) \\
& =\frac{1}{2} E\left|a_{1} \boldsymbol{c}_{n}^{\dagger} \boldsymbol{B} \boldsymbol{c}_{0}+\boldsymbol{c}_{n}^{\dagger} \boldsymbol{B} \boldsymbol{w}_{\boldsymbol{I}}\right|^{2} \\
& =\frac{1}{2} a_{1}^{2} E\left|\boldsymbol{c}_{n}^{\dagger} \boldsymbol{B} \boldsymbol{c}_{0}\right|^{2}+\frac{1}{2} E\left|\boldsymbol{c}_{n}^{\dagger} \boldsymbol{B} \boldsymbol{w}_{\boldsymbol{I}}\right|^{2} \\
& =\frac{1}{2} a_{1}^{2} E\left(\boldsymbol{c}_{n}^{\dagger} \boldsymbol{B} \boldsymbol{c}_{0} \boldsymbol{c}_{0}^{\dagger} \boldsymbol{B} \boldsymbol{c}_{n}\right)+\frac{1}{2} E\left(\boldsymbol{c}_{n}^{\dagger} \boldsymbol{B} \boldsymbol{w}_{\boldsymbol{I}} \boldsymbol{w}_{\boldsymbol{I}}^{\dagger} \boldsymbol{B} \boldsymbol{c}_{n}\right) \\
& \stackrel{(a)}{=}\left(\frac{a_{1}^{2}}{2}+\sigma_{\boldsymbol{I}}^{2}\right) E\left(\boldsymbol{c}_{n}^{\dagger} \boldsymbol{B}^{2} \boldsymbol{c}_{n}\right) \\
& \stackrel{(b)}{=}\left(\frac{a_{1}^{2}}{2}+\sigma_{\boldsymbol{I}}^{2}\right) E\left(\boldsymbol{c}_{n}^{\dagger} \boldsymbol{B} \boldsymbol{c}_{n}\right) \\
& =\left(\frac{a_{1}^{2}}{2}+\sigma_{\boldsymbol{I}}^{2}\right) E\left(\sum_{i, j} b_{i j} \boldsymbol{c}_{n, i} \boldsymbol{c}_{n, j}\right) \\
& =\left(\frac{a_{1}^{2}}{2}+\sigma_{\boldsymbol{I}}^{2}\right) \sum_{i, i} b_{i i}=\left(\frac{a_{1}^{2}}{2}+\sigma_{\boldsymbol{I}}^{2}\right) \operatorname{trace}(\boldsymbol{B}) \\
& =\left(\frac{a_{1}^{2}}{2}+\sigma_{\boldsymbol{I}}^{2}\right) d_{N}
\end{aligned}
$$

where (a) follows from our heuristic assumption, and (b) is due to Lemma 1 . Note that the factor of two in $a_{1}^{2} / 2$ would not be present when we have real spreading sequences. Similarly, for $n \neq m$ and $n, m \neq n_{1}$

$$
\begin{aligned}
\operatorname{Cov}\left(X_{n}, X_{m}\right)= & \frac{1}{2} E\left(X_{n} X_{m}^{\star}\right) \\
= & \frac{1}{2} a_{1}^{2} E\left(\boldsymbol{c}_{n}^{\dagger} \boldsymbol{B} \boldsymbol{c}_{0} \boldsymbol{c}_{0}^{\dagger} \boldsymbol{B} \boldsymbol{c}_{m}\right) \\
& +\frac{1}{2} E\left(\boldsymbol{c}_{n}^{\dagger} \boldsymbol{B} \boldsymbol{w}_{\boldsymbol{I}} \boldsymbol{w}_{\boldsymbol{I}}^{\dagger} \boldsymbol{B} \boldsymbol{c}_{m}\right) \\
= & \left(\frac{a_{1}^{2}}{2}+\sigma_{\boldsymbol{I}}^{2}\right) E\left(\boldsymbol{c}_{n}^{\dagger} \boldsymbol{B} \boldsymbol{c}_{m}\right)=0 .
\end{aligned}
$$




\section{B. Detection Statistics}

In this case, the delay hypothesis is $\tau=n_{1}$ and the corresponding AML metric is

$$
X_{n_{1}}=a_{1} \boldsymbol{c}_{0}^{\dagger} \boldsymbol{B} \boldsymbol{c}_{0}+\boldsymbol{c}_{0}^{\dagger} \boldsymbol{B} \boldsymbol{w}_{\boldsymbol{I}}
$$

Consequently

$$
m_{d}=E\left(X_{n_{1}}\right)=a_{1} E\left(\boldsymbol{c}_{0}^{\dagger} \boldsymbol{B} \boldsymbol{c}_{0}\right)=a_{1} \operatorname{trace}(\boldsymbol{B})=a_{1} d_{N} \cdot
$$

To compute the variance of $X_{n_{1}}$, note that

$$
E\left(\left|X_{n_{1}}\right|^{2}\right)=a_{1}^{2} E\left|\boldsymbol{c}_{0}^{\dagger} B c_{0}\right|^{2}+E\left|c_{0}^{\dagger} B \boldsymbol{w}_{\boldsymbol{I}}\right|^{2}
$$

As before, we have $E\left|\boldsymbol{c}_{0}^{\dagger} \boldsymbol{B} \boldsymbol{w}_{\boldsymbol{I}}\right|^{2}=E\left(\left|\boldsymbol{c}_{0}^{\dagger} \boldsymbol{B} \boldsymbol{w}_{\boldsymbol{I}}\right|^{2} \mid \boldsymbol{B}\right)=$ $2 \sigma_{I}^{2} d_{N} ;$ and

$$
\begin{aligned}
& E\left(\left|\boldsymbol{c}_{0}^{\dagger} \boldsymbol{B} \boldsymbol{c}_{0}\right|^{2} \mid \boldsymbol{B}\right) \\
& \quad=E\left(\sum_{i, j, k, l} b_{i j} b_{k l}^{\star} c_{0, i} c_{0, j}^{\star} c_{0, k} c_{0, l}^{\star}\right) \\
& \quad=2 \sum_{i \neq j}\left|b_{i j}\right|^{2}+\sum_{i, k} b_{i i} b_{k k} \\
& \quad=2 \sum_{i, j}\left|b_{i j}\right|^{2}-2 \sum_{i} b_{i i}^{2}+\sum_{i, k} b_{i i} b_{k k} \\
& \quad \stackrel{(a)}{=} 2 \sum_{i} b_{i i}+\left(\sum_{i} b_{i i}\right)^{2}-2 \sum_{i} b_{i i}^{2} \\
& \quad=2 d_{N}+d_{N}^{2}-2 \sum_{i} b_{i i}^{2}
\end{aligned}
$$

where (a) is due to the fact that $B B^{\dagger}=B \Rightarrow b_{i i}=\sum_{j}\left|b_{i j}\right|^{2}$. It follows that:

$$
\begin{aligned}
\operatorname{Var}\left(X_{n_{1}} \mid \boldsymbol{B}\right) & =a_{1}^{2} \operatorname{Var}\left(\boldsymbol{c}_{0}^{\dagger} \boldsymbol{B} \boldsymbol{c}_{0}\right)+2 \sigma_{\boldsymbol{I}}^{2} d_{N} \\
& =2 a_{1}^{2} d_{N}-2 a_{1}^{2} \sum_{i=1}^{M N} b_{i i}^{2}+2 \sigma_{\boldsymbol{I}}^{2} d_{N}
\end{aligned}
$$

Using the Cauchy-Schwarz inequality, we have

$$
\begin{aligned}
\left(\sum_{i=1}^{M N} b_{i i}\right)^{2} & \leq M N \sum_{i=1}^{M N} b_{i i}^{2} \\
\Rightarrow \sigma_{d}^{2}=\frac{1}{2} \operatorname{Var}\left(X_{n_{1}}\right) & \leq a_{1}^{2} d_{N}-a_{1}^{2} \frac{d_{N}^{2}}{M N}+\sigma_{I}^{2} d_{N} \\
& =\left(\frac{a_{1}^{2} d_{S}}{M N}+\sigma_{\boldsymbol{I}}^{2}\right) d_{N} .
\end{aligned}
$$

We note that a weaker version of the above bound was derived independently and in a completely different context in [22]. Finally, for $n \neq n_{1}$

$$
\begin{aligned}
\operatorname{Cov}\left(X_{n}, X_{n_{1}}\right) & =\frac{1}{2} E\left(X_{n} X_{n_{1}}^{\star}\right) \\
& =\frac{1}{2} a_{1}^{2} E\left(\boldsymbol{c}_{n}^{\dagger} \boldsymbol{B} \boldsymbol{c}_{0} \boldsymbol{c}_{0}^{\dagger} \boldsymbol{B} \boldsymbol{c}_{0}\right)
\end{aligned}
$$

$$
\begin{aligned}
& +\frac{1}{2} E\left(\boldsymbol{c}_{n}^{\dagger} \boldsymbol{B} \boldsymbol{w}_{\boldsymbol{I}} \boldsymbol{w}_{\boldsymbol{I}}^{\dagger} \boldsymbol{B} \boldsymbol{c}_{0}\right) \\
= & \frac{a_{1}^{2}}{2} E\left(\boldsymbol{c}_{n}^{\dagger} \boldsymbol{B} \boldsymbol{c}_{0} \boldsymbol{c}_{0}^{\dagger} \boldsymbol{B} \boldsymbol{c}_{0}\right) \\
= & \frac{a_{1}^{2}}{2} \sum_{i, j, k, l} E\left(b_{i j} b_{k l l} c_{0, i} c_{0, j}^{\star} c_{0, k} c_{n, l}^{\star}\right)=0
\end{aligned}
$$

since $\boldsymbol{c}_{n}$ is zero mean and independent of $\boldsymbol{c}_{0}$.

\section{REFERENCES}

[1] S. Bensley and B. Aazhang, "Subspace-based channel estimation for CDMA system," IEEE Trans. Commun., vol. 44, pp. 1009-1020, Aug. 1996.

[2] E. G. Storm et al., "Propagation delay estimation in asynchronous DS-CDMA systems," IEEE Trans. Commun., vol. 44, pp. 84-93, Jan. 1996.

[3] D. Zhang et al., "An efficient code-timing estimator for DS-CDMA signals," IEEE Trans. Signal Processing, vol. 45, pp. 82-89, Jan. 1997.

[4] S. Bensley and B. Aazhang, "Maximum-likelihood synchronization of a single user for CDMA systems," IEEE Trans. Commun., vol. 46, pp. 392-399, Mar. 1998.

[5] U. Madhow, "Blind adaptive interference suppression for near-far resistant acquisition and demodulation of DS-CDMA signals," IEEE Trans. Signal Processing, vol. 45, pp. 124-36, Jan. 1997.

[6] _ - "Blind adaptive interference suppression for direct-sequence CDMA,” Proc. IEEE, vol. 86, pp. 2049-2069, Oct. 1998.

[7] S. Vembu and A. J. Viterbi, "Two different philosophies in CDMA-A comparison," in Proc. IEEE 46th Veh. Technol. Conf., Atlanta, GA, Apr. 1996, pp. 869-73.

[8] V. V. Veeravalli, "The role of coding in CDMA systems with multiuser detection," in Proc. 1997 ICPWC, Mumbai, India, Dec. 1997.

[9] S. Parkvall, "User performance variability in DS-CDMA systems-Long vs short spreading sequences," in Proc. 1998 GLOBECOM, Sydney, Australia, Nov. 1998.

[10] TIA/EIA/IS-95, Mobile Station-Base Station Compatibilty Standard for Dual-Mode Wideband Spread Spectrum Cellular System: Telecommun. Industry Assoc., July 1993.

[11] TIA cdma.2000, Wideband cdmaOne Radio Transmission Technology Proposal: Int. Telecommun. Union, Radiocommun. Study Groups, June 1998.

[12] R. Cameron and B. Woerner, "Synchronization of CDMA systems employing interference cancellation," in Proc. VTC 1996, 1996, pp. $178-182$.

[13] R. Lupas and S. Verdu, "Near-far resistance of multi-user detectors in asynchronous channels," IEEE Trans. Commun., vol. 38, pp. 496-508, Apr. 1990.

[14] R. A. Iltis and L. Mailender, "Multiuser code acquisition using parallel decorrelators," in Proc. 1994 CISS, Princeton, NJ, Mar. 1994.

[15] A. Mantravadi and V. Veeravalli, "On discrete sufficient statistics for detection asynchronous band-limited CDMA systems," in Proc. 1999 CISS. Baltimore, MD, Mar. 1999.

[16] G. Golub and C. F. Van Loan, Matrix Computations. Baltimore, MD: John Hopkins University Press, 1996.

[17] A. Mantravadi and V. Veeravalli, "On discrete sufficient statistics for acquisition in asynchronous band-limited CDMA systems," in Proc. Alletron 99, Monticello, IL, Sept. 1999.

[18] J. M. Holtzman, "A simlpe, accurate method to calculate SSMA error probabilities," IEEE Trans. Commun., vol. 49, pp. 461-464, Mar. 1992.

[19] S. Verdu and S. Shamai, "Spectral efficiency of CDMA with random spreading," IEEE Trans. Inform. Theory, vol. 45, pp. 622-640, Mar. 1999.

[20] D. H. Johnson and D. E. Dudgeon, Array Signal Processing. Englewood Cliffs, NJ: Prentice-Hall, 1993.

[21] A. Mantravadi and V. Veeravalli, "Multi-access interference resistant acquisition for CDMA systems with long spreading sequences," in Proc. 1998 CISS, Princeton, NJ, Mar. 1998, pp. 141-146.

[22] D. Tse and S. Hanly, "Linear multiuser receivers: Effective interference, effective bandwidth and user capacity," IEEE Trans. Inform. Theory, pp. 641-657, Mar. 1999. 


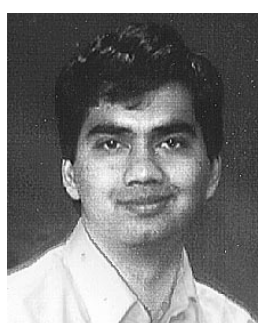

Ashok Mantravadi received the B.Tech degree in electronics and communications engineering from the Indian Institute of Technology, Madras in 1996, and the M.S degree from the School of Electrical Engineering at Cornell University in January, 1999. Between June and August 1997, he worked as an Engineering Intern with the the CDMA system design group at Qualcomm Inc, San Diego. He is currently in the the $\mathrm{Ph} . \mathrm{D}$ program at Cornell University, where he has been a recipient of the John McMullen and Schlumberger Fellowships. His research interests include spread spectrum systems, wireless multiuser communications and space-time signal processing.

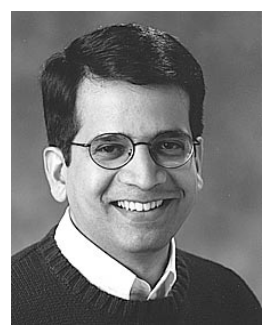

Venugopal V. Veeravalli (S'86-M'92-SM'98) received the Ph.D. degree in 1992 from the University of Illinois at Urbana-Champaign, the M.S. degree in 1987 from Carnegie-Mellon University, Pittsburgh, $\mathrm{PA}$, and the B.Tech. degree in 1985 from the Indian Institute of Technology, Bombay, (Silver Medal Honors), all in electrical engineering.

$\mathrm{He}$ joined the University of Illinois at Urbana-Champaign in 2000, where he is currently an Assistant Professor in the Department of Electrical and Computer Engineering, and a Research Associate Professor in the Coordinated Science Laboratory. He was a Postdoctoral Fellow at Harvard University, Cambridge, MA, during 1992-1993, an Assistan Professor at the City College of NY during 1993-1994, a Visiting Assistant Professor at Rice University, Houston, TX, during 1994-1996, and an Assistant Professor at Cornell University, Ithaca, NY, during 1996-2000. His research interests include mobile and wireless communications, detection and estimation theory, and information theory.

Dr. Veeravalli is currently an Associate Editor for IEEE JOURNAL ON Selected AREas in Communications-Wireless Communication Series, and an Editor for Communications in Information and Systems (CIS). Among the awards he has received for research and teaching are the IEEE Browder J. Thompson Best Paper Award in 1996, the National Science Foundation CAREER Award in 1998, the Presidential Early Career Award for Scientists and Engineers (PECASE) in 1999, and the Michael Tien Excellence in Teaching Award from the College of Engineering, Cornell University in 1999. 